\title{
Can we detect Galactic spiral arms? 3D dust distribution in the Milky Way
}

\section{Sara Rezaei Kh., Coryn A. L. Bailer-Jones, Morgan Fouesneau and Richard Hanson}

Max Planck Institute for Astronomy (MPIA), Königstuhl 17, 69117 Heidelberg, Germany

email: sara@mpia.de

\begin{abstract}
We present a model to map the 3D distribution of dust in the Milky Way. Although dust is just a tiny fraction of what comprises the Galaxy, it plays an important role in various processes. In recent years various maps of dust extinction have been produced, but we still lack a good knowledge of the dust distribution. Our presented approach leverages line-of-sight extinctions towards stars in the Galaxy at measured distances. Since extinction is proportional to the integral of the dust density towards a given star, it is possible to reconstruct the 3D distribution of dust by combining many lines-of-sight in a model accounting for the spatial correlation of the dust. Such a technique can be used to infer the most probable 3D distribution of dust in the Galaxy even in regions which have not been observed. This contribution provides one of the first maps which does not show the "fingers of God" effect. Furthermore, we show that expected high precision measurements of distances and extinctions offer the possibility of mapping the spiral arms in the Galaxy.
\end{abstract}

Keywords. Stars: Parallaxes - Stars: Distances - Galaxy: Dust Map - Galaxy: Milky Way ISM: Dust - ISM: Extinction

\section{Introduction}

Dust is a tiny fraction of the mass of a galaxy; yet it plays an important role in different processes in the Galaxy; from star and planet formation and evolution to obscuring stellar lights via extinction. Therefore it is key to studying the different properties of this Interstellar Medium (ISM) component, especially its 3D distribution in the Galaxy.

Many studies have mapped (partially) the distribution of the extinction in the Galaxy using various approaches; from early 2D maps (e.g. Schlegel et al. 1998) to more recent 3D maps (e.g. Hanson \& Bailer-Jones 2014, Hanson et al. 2016, Marshall et al. 2006, Sale et al. 2014, Green et al. 2015, Lallement et al. 2014). Looking in more detail, most of these maps show a common caveat: there are some lines-of-sight (l.o.s) traces, so-called "fingers of god", arising from the fact that each l.o.s is treated independently.

As published in Rezaei Kh. et al. 2017, we present a new method, which explicitly takes into account the correlations between close by l.o.s; therefore removing any l.o.s effect. Furthermore, we map the dust density distribution in the Galaxy rather than the extinction; while extinction is the integral over the dust densities along the l.o.s towards a star, dust density is the local property of the ISM. This way we present one of the first $3 \mathrm{D}$ dust maps in the Galaxy with no "fingers of god" effect.

\section{Testing the method with mock data}

Our method uses 3D positions and extinctions of stars within the Galaxy to infer the dust density at arbitrary positions in the $3 \mathrm{D}$ space. To do so, we divide each l.o.s into 


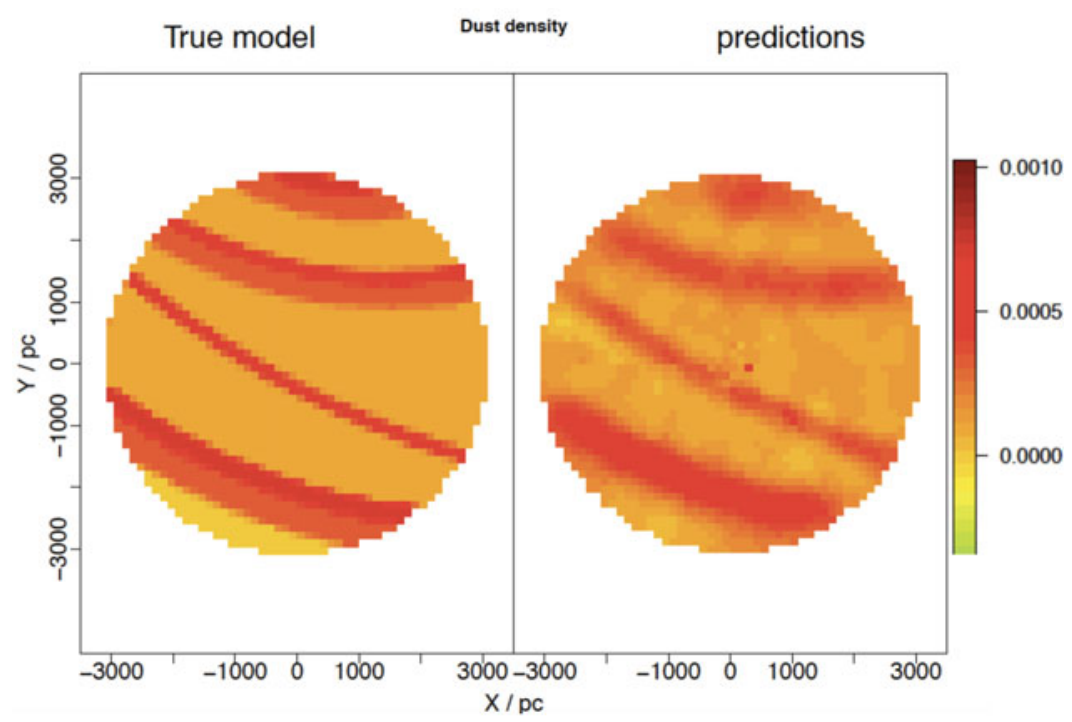

Figure 1. Comparing mock distribution (left) with model predictions (right) of dust density in the Galactic disk $\left(b<2^{\circ}\right)$. Sun is at $(0,0)$ and from the Sun upwards is toward the Galactic centre. Color indicates the dust density values in units of degree of attenuation per parsec.

small 1D cells and combine them with a Gaussian process, which exploits the correlation between these cells: the closer two points in the Galaxy in $3 \mathrm{D}$, the more correlated they are. Through this method plus some linear algebra (see Rezaei Kh. et al. 2017), we find the posterior PDF of the dust density at any position in the Galaxy.

We test our model on a fiducial dust density similar to the disk of our Galaxy, i.e. overdensities at the locations of the spiral arms. We simulate 8000 stars at random positions in the disk of the Galaxy (2 degrees in latitude) and out to $3 \mathrm{kpc}$, generate their extinctions based on our mock model and add random Gaussian extinction noise with $\sigma=0.05$ mag. We use these mock dataset as our input data and ask our model to find the dust density in the disk which describes best these noisy extinction measurements.

Figure 1 compares the predicted dust density (right) with the "true" distribution (left) used to generate these data. Qualitatively, the predictions recover all intrinsic structures, and the model precisely predicts the location and over-densities of the spiral arms.

\section{Application on the real data}

In this section, we infer the dust density in the Galaxy based on two different sets of observations: first, the APOGEE red clump (RC) star sample from Bovy et al. 2014, and second, the Gaia TGAS catalogue (Gaia Collaboration et al. 2016).

\subsection{Dust map in the disk with APOGEE RC stars}

Bovy et al. 2014 provides a catalogue of Red Clump stars based on APOGEE data. From their sample we select $\sim 8000$ stars in the disk of the Galaxy (disk height $= \pm 500 \mathrm{kpc}$ ) and out to $5 \mathrm{kpc}$ from the Sun with distances and K-band extinction $\left(A_{k}\right)$ values. Their typical uncertainty in extinction is $\sigma_{A_{k}}=0.05 \mathrm{mag}$ which we use in our model. However, we neglect the $5 \%$ uncertainty in the distance.

We compare dust features that may be associated with the spiral arms in our Galaxy described by the model of Reid et al. 2014, which assign high-mass star forming regions (masers) to the spiral arms based on their longitude-velocity diagram. We over-plot their 


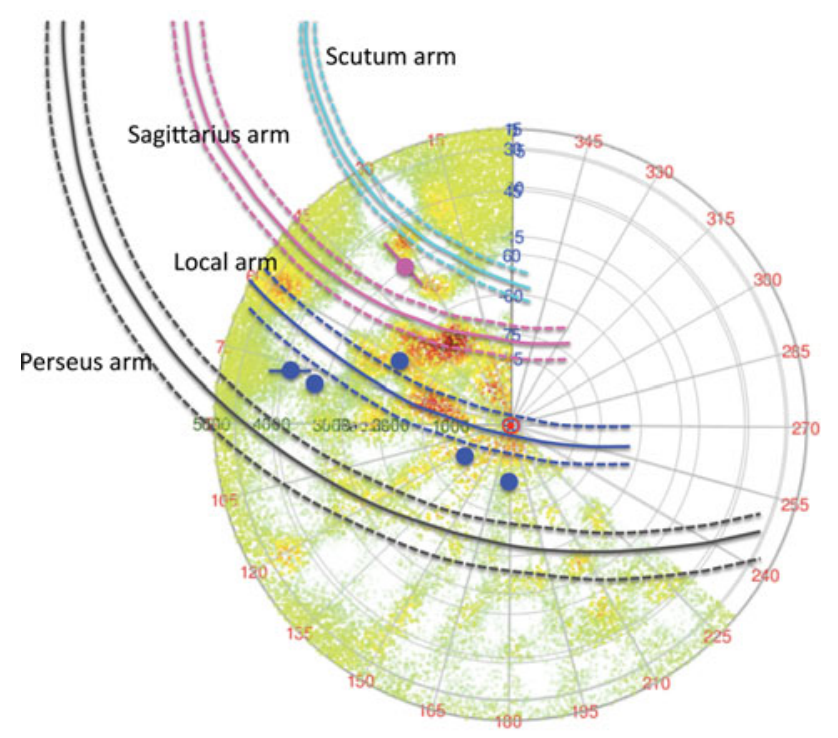

Figure 2. Dust density predictions in the Galactic disk, over-plotted with the spiral arm model and maser emissions from Reid et al. 2014. Color coding is as in fig. 2. Blue/pink circles are masers.

model of spiral arms plus some of their associated masers on our dust map as seen in Fig. 2. Some of our dust clouds (red) lie within $1 \sigma$ of the arms; especially near the Sagittarius and the Local arms. However, some other dust clouds and masers from Reid et al. 2014 don't seem associated with the current model, for instance we do not find high dust density near the Perseus arm. We need more data with higher precision for further discussion on the Galactic spiral arms. Looking at fig. 2, there are some negative dust densities which are unphysical but due to the Gaussian nature of our model. This can be improved by truncating the Gaussian distribution or using other positive distributions.

\section{2. local 3D dust map with Gaia TGAS}

The higher the data quality, the more precisely can we map the dust. Therefore we explored the Gaia TGAS data. TGAS distances are provided by Astraatmadja and BailerJones 2016 using Bayesian inference to infer distances from Gaia parallaxes, and we derive extinctions based on Rayleigh Jeans Color Excess (RJCE; Majewski et al. 2011) similarly to the extinctions in APOGEE (Zasowski et al. 2013), though restricting ourselves to giant stars where it was calibrated. As we currently neglect distance uncertainties in our model, we select stars with distance/standard error $>10$. This gives us $\sim 4000$ stars and only out to $500 \mathrm{pc}$ as input to map the dust density for a volume of 500 pc radius.

Figure 3 shows a top-down view of the 3D plot of dust density predictions, where the Sun is at the centre. We see some over-density between longitudes of $100^{\circ}$ to $115^{\circ}$ in the Galactic disk and at around $300-450$ pc. There are also some other over-densities like the one at negative latitude of $\sim-15^{\circ}$ and longitude of $\sim 200^{\circ}$ at $\sim 400 \mathrm{pc}$ which could be associated with the Orion cloud (Schlafly et al. 2014).

As discussed already, we need to use more data to better constrain the 3D structure of the dust in our Galaxy, which will eventually come from the next Gaia data releases and would enable us to build a precise 3D map of dust in the Milky way with no l.o.s. effect and will try to find some traces of spiral arms in our Galaxy. 


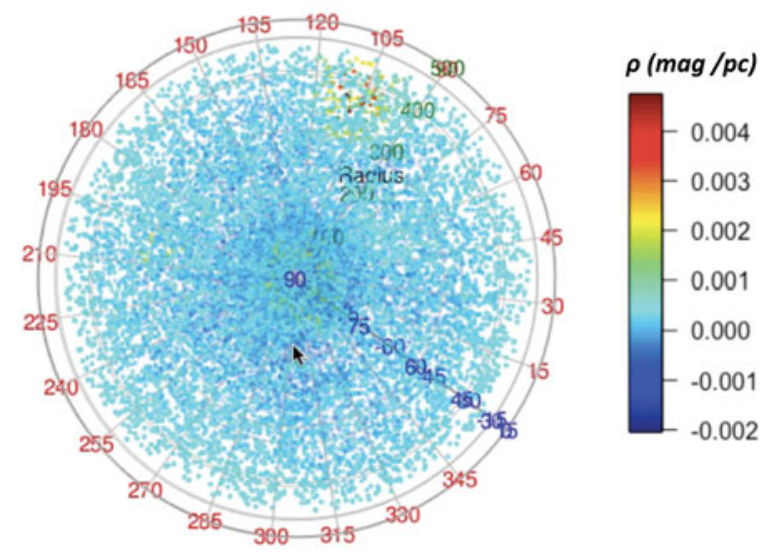

Figure 3. Top-down view of the 3D dust density predictions in a local 500 pc volume using TGAS data. Sun is in the centre and red numbers define the longitudes.

\section{Acknowledgments}

This work has made use of data from the European Space Agency (ESA) mission Gaia (http://www.cosmos.esa.int/gaia), processed by the Gaia Data Processing and Analysis Consortium (DPAC, http://www.cosmos.esa.int/web/gaia/dpac/consortium). Funding for the DPAC has been provided by national institutions, in particular the institutions participating in the Gaia Multilateral Agreement.

\section{References}

Astraatmadja, T. L. \& Bailer-Jones, C. A. L. 2016, ApJ, 833, 119

Bovy, J., Nidever, D. L., Rix, H.-W. et al. 2014, ApJ, 790, 127

Gaia Collaboration et al. 2016, A\&A, 595, A2

Green, G. M., Schlafly, E. F., Finkbeiner, D. P. et al. 2015, ApJ, 810, 25

Hanson, R. J. \& Bailer-Jones, C. A. L. 2014, MNRAS, 438, 2938

Hanson, R. J., Bailer-Jones, C. A. L., Burgett, W. S. et al. 2016, MNRAS, 463, 3604

\{Lallement, R., Vergely, J.-L., Valette, B., Puspitarini, L., Eyer, L., \& Casagrande, L. 2014, $A \mathscr{G} A, 561, \mathrm{~A} 91$

Majewski, S. R., Zasowski, G., \& Nidever, D. L. 2011, ApJ, 739, 25

Marshall, D. J., Robin, A. C., Reyl, C., Schultheis, M., \& Picaud, S. 2006, A\&\&A, 453, 635

Reid, M. J., Menten, K. M., Brunthaler, A. et al. 2014, ApJ, 783, 130

Rezaei Kh., S., Bailer-Jones, C. A. L., Hanson, R. J., \& Fouesneau, M. 2017, A\&3A, 598, A125

Sale, S. E., Drew, J. E., Barentsen, G. et al. 2014, MNRAS, 443, 2907

Schlafly, E. F., Green, G., Finkbeiner, D. P. et al. 2014, ApJ, 786, 29

Schlegel, D. J., Finkbeiner, D. P., \& Davis, M. 1998, ApJ, 500, 525

Zasowski, G., Johnson, J. A., \& Frinchaboy, P. M. 2013, AJ, 146, 81 